\title{
New Results on the Composition of the Outer Planets and Titan
}

\author{
Thierry Fouchet \\ Lesia - Université Pierre et Marie Curie, Observatoire de Meudon, 5 \\ Place Jules Janssen, 92195 Meudon Cedex
}

\begin{abstract}
In this brief summary, I present recent progress on our knowledge of the Giant Planets and Titan atmospheric composition, as well as the impact of this progress on our understanding of Solar System formation, and atmospheric chemistry.
\end{abstract}

\section{Isotopic composition}

\subsection{Deuterium}

The $\mathrm{D} / \mathrm{H}$ ratio in Solar System objects is a powerful tool to investigate the formation of planetary objects in the proto-solar nebula. However, most determinations of $\mathrm{D} / \mathrm{H}$ in Giant Planets suffered from uncertainties, due to observational difficulties, or due to the isotopic fractionation existing between HD and $\mathrm{CH}_{3} \mathrm{D}$. The fractionation coefficient $f$, that depends on reaction kinetics and atmospheric dynamical time scale, is not precisely known.

Recently, ISO detected for the first time the rotational lines of HD . This new situation allowed Feuchtgruber et al. (1999) and Lellouch et al. (2001) a simple and coherent determination of $\mathrm{D} / \mathrm{H}$ on the four Giant Planets. The results $\left(\mathrm{D} / \mathrm{H}=(2.25 \pm 0.35) \times 10^{-5}\right.$ for Jupiter, $\left(1.70_{-0.45}^{+0.75}\right) \times 10^{-5}$ for Saturn, $\left(5.50_{-1.5}^{+3.5}\right) \times 10^{-5}$ for Uranus, and $\left(6.5_{-1.5}^{+2.5}\right) \times 10^{-5}$ for Neptune), confirmed that Uranus and Neptune have been enriched in D during their formation, by mixing of their atmosphere with a large, D-rich, icy core. Assuming complete mixing, it is possible to retrieve the $\mathrm{D} / \mathrm{H}$ ratio in the ices that formed the cores : $\mathrm{D} / \mathrm{H}=\left(8.5_{-3.8}^{+10.0}\right) \times 10^{-5}$. This lower $\mathrm{D} / \mathrm{H}$ than measured in comets bears important implications for the dynamics of the proto-solar nebula.

\subsection{Nitrogen}

Different studies, either using remote sensing spectroscopy with ISO (Fouchet et al. 2000a), or in-situ measurements (Owen et al. 2001), have shown that the ${ }^{15} \mathrm{~N} /{ }^{14} \mathrm{~N}$ ratio in Jupiter is significantly lower in the Jovian atmosphere than on the Earth: $(2.20 \pm 0.40) \times 10^{-3}$ compared to $3.67 \times 10^{-3}$. As Jupiter has not been isotopically fractionated, the Jovian ratio is similar to the solar ${ }^{15} \mathrm{~N} /{ }^{14} \mathrm{~N}$. In contrast, the comets, the Earth, Mars, and Titan have been fractionated either during their formation or during their subsequent evolution. This situation is especially of great value to understand the origin of terrestrial nitrogen, which still remains unclear. 


\section{Photochemistry}

Photochemistry in the Giant Planets is triggered by $\mathrm{CH}_{4}$ photolysis, and leads to the formation of heavier hydrocarbons, principally $\mathrm{C}_{2} \mathrm{H}_{6}$ and $\mathrm{C}_{2} \mathrm{H}_{2}$. The atmospheric dynamics redistributes the product vertically and horizontally. Recently, Fouchet et al. (2000b) and Nixon et al. (2002) observed for the first time the vertical and meridional variations of $\mathrm{C}_{2} \mathrm{H}_{6}$ and $\mathrm{C}_{2} \mathrm{H}_{2}$. The results show that $\mathrm{C}_{2} \mathrm{H}_{2}$ abundance decreases faster than $\mathrm{C}_{2} \mathrm{H}_{6}$ abundance with increasing pressures and latitudes. This result backs photochemical models that predicted a longer lifetime for ethane than for acetylene.

In contrast, photochemical models have a harder task at predicting the abundance of trace hydrocarbons. Bézard et al. (2001a) observed a $\mathrm{C}_{2} \mathrm{H}_{4}$ column abundance of $6 \times 10^{14}$ on Jupiter and $3.5 \times 10^{15} \mathrm{~cm}^{-2}$ on Saturn, approximately $30 \%$ less than predicted. For benzene, detected by Bézard et al. (2001b) on Jupiter and Saturn by ISO, the column abundances $\left[(1.5-13.5) \times 10^{14}\right.$ and $\left(3.6^{-}\right.$ $6.8) \times 10^{13} \mathrm{~cm}^{-2}$ ] are $50 \%$ lower than expected.

\section{Oxygen in the outer Solar System}

Recently, ISO detected water in the stratosphere of the four giant planets and Titan (Feuchtgruber et al. 1997, Coustenis et al. 1998, Moses et al. 2000, Lellouch et al. 2002). Water cannot be transported from the underlying levels, has it must condense out at the very low tropopause temperatures. Instead, it must come from an exogenic source. On Jupiter, Lellouch et al. (2002) identified the source as, most likely, the SL9 collision. Moreover Lellouch et al. put an upper limit to the flux of water due to IDPs at Jupiter : $8 \times 10^{4} \mathrm{~cm}^{-2} \mathrm{~s}^{-1}$. On Saturn and Titan the exogenic water flux is higher than this upper limit (respectively $(4 \pm 2) \times 10^{6}$, and $\left.(0.8-2.6) \times 10^{6} \mathrm{~cm}^{-2} \mathrm{~s}^{-1}\right)$. Thus, IDPs do not constitute a realistic source of the Saturnian System. Instead, sputtering of the rings for Saturn, and from Enceladus for Titan appears as possible origins. For Uranus and Neptune, the O flux (respectively $(0.6-1.6) \times 10^{5}$, and $(1.2-150) \times$ $10^{5} \mathrm{~cm}^{-2} \mathrm{~s}^{-1}$ ), could be best explained by IDPs precipitation, the larger flux observed at Neptune being due to its proximity to the Kuiper belt.

Using ground-based spectroscopy, Bézard et al. (2002) showed that CO is present in Jupiter troposphere, but also in its stratosphere, highlighting the existence of both the internal and exogenic CO sources. The tropospheric abundance, $1.0 \pm 0.2 \mathrm{ppb}$, is consistent with recent chemical and dynamical calculation. The external source cannot be explained entirely by the SL9 event, but requires an additional external $\mathrm{CO}$ flux. The authors concluded that the most likely source is the in-fall of a kilometer-sized body each century.

\section{References}

Bézard, B. et al. 2001a, BAAS, 33, 1079

Bézard, B. et al. 2001b, Icarus, 154, 495

Bézard, B. et al. 2002, Icarus, 159, 95

Coustenis, A. et al. 1998, A\&A, 336, L85

Feuchtgruber, H. et al. 1997, Nature, 389, 159 
Feuchtgruber, H. et al. 1999, A\&A, 341, L17

Fouchet, T. et al. 2000a, Icarus, 143, 223

Fouchet, T. et al. 2000b, A\&A, 355, L13

Lellouch, E. et al. 2001, A\&A, 370, 610

Lellouch, E. et al. 2002, Icarus, 159, 112

Moses, J. et al. 2000, Icarus, 145, 166

Nixon, C. et al. 2002, BAAS, 34, 851

Owen, T. et al. 2001, ApJ, 553, L77 